\title{
Massless scalar fields and topological black holes
}

\author{
Tekin Dereli and Yuri N. Obukhov* \\ Department of Physics, Middle East Technical University, 06531 Ankara, Turkey
}

file scalar.tex, 24 September 1999, draft

\begin{abstract}
The exact static solutions in the higher dimensional Einstein-Maxwell-KleinGordon theory are investigated. With the help of the methods developed for the effective dilaton type gauge gravity models in two dimensions, we find new spherically and hyperbolically symmetric solutions which generalize the four dimensional configurations of Dereli-Eriş. We show that, like in four dimensions, the non-trivial scalar field yields, in general, a naked singularity. The new solutions are compared with the higher dimensional Brans-Dicke black hole type solutions.
\end{abstract}

PACS no.: 04.50.+h; 04.20.Jb; 03.50.Kk

*On leave from: Department of Theoretical Physics, Moscow State University, 117234 Moscow, Russia 


\section{INTRODUCTION}

The study of exact solutions remains one of the central issues of Einstein's gravitational theory and its generalizations. Both the higher-dimensional and lower-dimensional gravity models (see, e.g., [四) have attracted considerable attention recently. This interest was partly motivated by the idea that the geometrical structures not confined to the dimension four may be helpful in understanding the four-dimensional physics, and partly was a due to the development of (super)unification approaches.

Topological black holes possess horizons with non-trivial topology. They naturally arise in a number of gravitational models [2 [4] (in particular, as black strings, black branes, etc) and have rather different properties from the usual black holes with spherical horizons. Higher dimensional generalizations of these object were considered recently in [5].

As it is well known, the non-trivial scalar field may, in general, destroy a horizon and produce a naked singularity instead. This fact, first noticed in four dimensions, cf. [6], was later confirmed also for the higher dimensions $[7,8]$, although for certain types of dilaton couplings the higher-dimensional black holes with scalar fields do exist [9,10]. For a comprehensive recent review see [1]].

It seems interesting to consider Einstein's gravity with all the three above mentioned features combined: i.e. in an arbitrary dimensional spacetime, with a scalar field, and possibly with non-trivial topology of horizons. This problem is studied in the present paper. Technically, we use the methods developed previously for the two-dimensional gauge gravity models [15,16]. For this purpose, in Sect. II, the original problem is reduced from $(d+n)$ dimensions to an effective $d$-dimensional model by means of the Kaluza-Klein scheme with an "internal" $n$-dimensional space of constant curvature (positive, negative or zero). Then, in Sect. [II, the case $d=2$ is analyzed in detail with the help of the technique developed in [15,16. The new exact solutions are derived in Sect. IV, and we discuss their properties and make conclusions in Sect. V. 


\section{KALUZA-KLEIN SCHEME}

Let us consider the Kaluza-Klein reduction of a $(d+n)$-dimensional manifold to the physical $d$-dimensional Riemannian spacetime $M^{d}$ with an $n$-dimensional internal space of constant curvature. Denote the components of the higher dimensional curvature two-form $\mathcal{R}^{A B}$ with respect to a local orthonormal frame $E_{A}$. The dual coframe one-forms are denoted $\vartheta^{A}$, and the indices run $A, B, \ldots=0,1, \ldots, d+n-1$. The general Kaluza-Klein reduction of an $(d+n)$-dimensional manifold with a compactification on an $n$-torus involves $n 1$-forms $A^{a}$ and $n^{2}$ scalar fields $\Phi_{b}^{a}$. Here we will consider a simplified scheme without the Kaluza-Klein 1-forms (gauge fields). Then the consistent decomposition of the metric reads:

$$
\stackrel{(d+n)}{g}=\stackrel{(d)}{g}+e^{-\frac{4}{n} \Phi \stackrel{(n)}{g} .}
$$

Here $\Phi$ is the Kaluza-Klein scalar field (only one scalar survives in absence of $A^{a}$ ) which depends only on the coordinates of $M^{d}$, and

$$
\begin{aligned}
& \stackrel{(d)}{g}=g_{\alpha \beta} \vartheta^{\alpha} \otimes \vartheta^{\beta}, \\
& \stackrel{(n)}{g}=g_{a b} \vartheta^{a} \otimes \vartheta^{b},
\end{aligned}
$$

describe, respectively, the metric of the physical spacetime $\left[\right.$ with $g_{\alpha \beta}=\operatorname{diag}(-1,1, \ldots, 1)$ as a $d$-dimensional Minkowski metric] and the internal space [with $g_{a b}=\delta_{a b}$ ] of a constant curvature $R^{a b}=-\lambda \vartheta^{a} \wedge \vartheta^{b}$. The constant $\lambda=+1$ for an $n$-sphere of a unit radius, $\lambda=0$ for flat space (e.g., hyperplane, cylinder or $n$-torus), and $\lambda=-1$ for a hyperbolic space.

The (local frame) indices clearly run: $\alpha, \beta, \ldots=0,1, \ldots, d-1$, and $a, b, \ldots=1, \ldots, n$.

The Einstein-Maxwell-Klein-Gordon theory with a cosmological term in $d+n$ dimensions reads

$$
L=-\frac{1}{2} \mathcal{R}^{A B} \wedge \eta_{A B}-\frac{1}{2} F \wedge \# F-\frac{1}{2}\left(d \phi \wedge \# d \phi+m^{2} \phi \# \phi\right)-\Lambda \eta .
$$

Here $F=d A$ is the Maxwell field strength two-form and $\phi$ is the scalar field. We are using the general notations and conventions of [14]. In particular, the Trautman's $\eta$-basis 
of exterior forms is defined by the Hodge duals of the products of coframe one-forms $\vartheta^{A}$ : given the volume $(d+n)$-form $\eta$, one has $\left.\left.\eta_{A}=\# \vartheta_{A}=E_{A}\right\rfloor \eta, \eta_{A B}=\#\left(\vartheta_{A} \wedge \vartheta_{B}\right)=E_{A}\right\rfloor \eta_{B}$, etc. Same notation is used for the lower-dimensional counterparts in $M^{d}$.

We will consider a massless Klein-Gordon field, so that $m^{2}=0$.

Assuming that the Maxwell and scalar fields are independent of the internal space coordinates, we straightforwardly obtain from (2.4) a dimensionally reduced Lagrangian:

$$
\begin{aligned}
L=e^{-2 \Phi}(- & \frac{1}{2} R^{\alpha \beta} \wedge \eta_{\alpha \beta}+2 \frac{n-1}{n} d \Phi \wedge * d \Phi+\frac{1}{2} \stackrel{(n)}{R} e^{\frac{4}{n} \Phi} \eta \\
& \left.-\frac{1}{2} F \wedge * F-\frac{1}{2} d \phi \wedge * d \phi-\Lambda \eta\right) .
\end{aligned}
$$

Here: $\stackrel{(n)}{R}=\lambda n(n-1)$ is the curvature scalar of the internal space, and from now on $\eta$ denotes the volume $d$-form and $*$ is the $d$-dimensional Hodge operator on $M^{d}$.

It seems worthwhile to note that Kaluza-Klein reduction from $(d+n)$ dimensions in the limit of $n \rightarrow \infty$ yields exactly the low-energy string model in an arbitrary dimension $d$ [21].

\section{CASE OF $D=2$ : EFFECTIVE TWO-DIMENSIONAL THEORY}

Let us put $d=2$. The above compactification obviously describes the general $2+n$ dimensional metric configurations with spherical $(\lambda=1)$, plane $(\lambda=0)$ and "hyperboloidal" $(\lambda=-1)$ symmetry. The reduced system (2.5) gives the dynamics of the "radial" variables in terms of a dilaton type gravity theory in two dimensions.

Recently there was an increasing interest in the so-called topological black holes which are defined as solutions of Einstein field equations for $\lambda=0,-1$.

We will look for the exact solutions of the Einstein-Maxwell-scalar field equations using the methods developed for the general two-dimensional Poincaré gauge gravity [15], see the review [16] which contains also a list of references to other approaches. In particular, as it is demonstrated in [16], any two-dimensional dilaton type model with a Lagrangian

$\eta\left[\mathcal{F}(\Phi) \widetilde{R}+\mathcal{G}(\Phi)\left(\partial_{\alpha} \Phi\right)^{2}+\mathcal{U}(\Phi)\right]$ can be recasted into a form of an effective theory in the 
Riemann-Cartan spacetime, and the gravitational field equations then can be straightforwardly integrated. The key role in this approach plays a two-dimensional torsion trace one-form $T$ which, together with the Hodge dual $* T$, provides a natural coframe basis in the spacetime.

For the Lagrangian (2.5) we have

$$
\mathcal{F}(\Phi)=\frac{1}{2} e^{-2 \Phi}, \quad \mathcal{G}(\Phi)=2\left(\frac{n-1}{n}\right) e^{-2 \Phi}, \quad \mathcal{U}(\Phi)=\frac{\lambda}{2} n(n-1) e^{-2\left(\frac{n-2}{n}\right) \Phi} .
$$

Correspondingly, one finds the Lagrangian of the equivalent Einstein-Cartan theory:

$$
L_{\mathrm{EC}}=-\frac{1}{2} \xi(\Phi) T^{\alpha} \wedge * T^{\alpha}-\frac{1}{2} \omega(\Phi) R^{\alpha \beta} \eta_{\alpha \beta}+\mathcal{U}(\Phi) \eta
$$

where

$$
\omega=e^{-2 \Phi}, \quad \xi=\left(\frac{n}{n-1}\right) e^{-2 \Phi} .
$$

Here $T^{\alpha}$ is the torsion two-form and the curvature is also constructed from the Lorentz connection with torsion. In two dimensions, the Hodge dual $t^{\alpha}=* T^{\alpha}$ is a (covector-valued) scalar. The torsion trace one-form $\left.T:=e_{\alpha}\right\rfloor T^{\alpha}=-t^{\alpha} \eta_{\alpha}$ together with $* T$ form a basis of the cotangent space when $t^{2}:=t^{\alpha} t_{\alpha}$ is non-zero. As a consequence, the two-metric arises as

$$
\stackrel{(2)}{g}=-\left(\vartheta^{0}\right)^{2}+\left(\vartheta^{1}\right)^{2}=\frac{1}{-t^{2}}\left[(T)^{2}-(* T)^{2}\right] .
$$

[Note that $t^{2}$ is a negative quantity]. The gravitational field equations, which arise from the variations of the action with respect to the coframe and connection one-forms (Palatini principle), after some rearrangements can be written as

$$
\begin{aligned}
d\left(\xi^{2} t^{2}\right) & =\left(\xi^{2} t^{2}-2 \xi \mathcal{U}\right) T+2 \xi S, \\
d(\xi * T) & =2 \mathcal{U} \eta+\vartheta^{\alpha} \wedge \Sigma_{\alpha}, \\
-2\left(\frac{n-1}{n}\right) d \Phi & =T .
\end{aligned}
$$

Here $S:=t^{\alpha} \Sigma_{\alpha}$, and as usual the source is represented by the energy-momentum one form which is obtained as a variational derivative of the matter Lagrangian [second line in (2.5)] with respect to the coframe: 


$$
\left.\left.\left.\Sigma_{\alpha}=-e^{-2 \Phi} \Lambda \eta_{\alpha}+\frac{1}{2} e^{-2 \Phi}\left(e_{\alpha}\right\rfloor F\right) * F+\frac{1}{2} e^{-2 \Phi}\left[\left(e_{a}\right\rfloor d \phi\right) * d \phi+d \phi\left(e_{a}\right\rfloor * d \phi\right)\right]
$$

From (3.4) and (3.7) we conclude that $\Phi$ can be taken as a local spatial coordinate, and one can construct a second leg of the coframe as

$$
\xi * T=B d \tau
$$

where $\tau$ is the local time coordinate, and $B=B(\tau, \Phi)$ is some yet unknown function.

The Maxwell equation $d\left(e^{-2 \Phi} * F\right)=0$ and the Klein-Gordon equation $d\left(e^{-2 \Phi} * d \phi\right)=0$ are easily integrated, yielding

$$
\begin{aligned}
& * F=Q e^{2 \Phi}, \\
& B \phi^{\prime}=c_{0},
\end{aligned}
$$

where $Q$ and $c_{0}$ are integration constants. Hereafter the prime denotes the derivative with respect to $\Phi$.

Substituting (3.9) and (3.10)-(3.11) into (3.8), and subsequently into (3.5)-(3.6), one finds a system of two differential equations for the unknown functions $\left(\xi^{2} t^{2}\right)$ and $B$ :

$$
\begin{aligned}
\left(\xi^{2} t^{2}\right)^{\prime} & =-2\left(\frac{n-1}{n}\right) \xi^{2} t^{2}+2 \lambda n(n-1) e^{-4\left(\frac{n-1}{n}\right) \Phi}-4 \Lambda e^{-4 \Phi}-2 Q^{2}+\frac{c_{0}^{2}}{2} \frac{\xi^{2} t^{2}}{B^{2}} \\
\frac{\left(B^{2}\right)^{\prime}}{2 B^{2}} & =\frac{2 \lambda n(n-1) e^{-4\left(\frac{n-1}{n}\right) \Phi}-4 \Lambda e^{-4 \Phi}-2 Q^{2}}{\xi^{2} t^{2}}
\end{aligned}
$$

It seems worthwhile to note that although most of the intermediate derivations were, strictly speaking, inapplicable to the case of $n=1$, the final system is meaningful also when $n=1$, yielding correct solutions.

Consider at first the case $n=2$ and $\lambda=1$. Then $\xi=2 e^{-2 \Phi}$ and the system (3.12)-(3.13) becomes

$$
\begin{aligned}
\frac{d\left(\xi^{2} t^{2}\right)}{d \Phi} & =-\xi^{2} t^{2}+2 \xi-\Lambda \xi^{2}-2 Q^{2}+\frac{c_{0}^{2}}{2} \frac{\xi^{2} t^{2}}{B^{2}} \\
\frac{1}{2 B^{2}} \frac{d B^{2}}{d \Phi} & =\frac{2 \xi-\Lambda \xi^{2}-2 Q^{2}}{\xi^{2} t^{2}} .
\end{aligned}
$$


All the $n \neq 2$ solutions can be generated from the solutions of (3.14)-(3.15) for the vanishing cosmological constant $\Lambda=0$. Indeed, in the general case let us introduce the new, rescaled, variables and constants:

$$
\begin{aligned}
& \widetilde{\Phi}=2\left(\frac{n-1}{n}\right) \Phi \quad \Longrightarrow \quad \widetilde{\xi}=2 e^{-2 \widetilde{\Phi}}=2 e^{-4\left(\frac{n-1}{n}\right) \Phi}, \\
& \left(\widetilde{\xi^{2} t^{2}}\right)=\frac{1}{\lambda}\left(\frac{2}{n}\right)^{2}\left(\xi^{2} t^{2}\right), \quad \widetilde{B^{2}}=\left(\frac{2}{n}\right)^{2} B^{2}, \\
& \widetilde{Q^{2}}=\frac{2}{n(n-1)} \frac{Q^{2}}{\lambda}, \quad \widetilde{c^{2}}=\frac{2}{n(n-1)} c_{0}^{2} .
\end{aligned}
$$

Clearly, we assume that $\lambda \neq 0$, the case $\lambda=0$ will be considered separately. Note that the quantities with tilde are not necessarily positive and this is an essential difference between the cases of $\lambda= \pm 1$. Substituting (3.16)-(3.18) into (3.12)-(3.13), we get

$$
\begin{aligned}
\frac{d\left(\widetilde{\xi^{2} t^{2}}\right)}{d \widetilde{\Phi}} & =-\left(\widetilde{\xi^{2} t^{2}}\right)+2 \widetilde{\xi}-2 \widetilde{Q^{2}}+\frac{\widetilde{c^{2}}}{2} \frac{\left(\widetilde{\xi^{2} t^{2}}\right)}{\widetilde{B}^{2}}, \\
\frac{1}{2 \widetilde{B^{2}}} \frac{d \widetilde{B^{2}}}{d \widetilde{\Phi}} & =\frac{2 \widetilde{\xi}-2 \widetilde{Q^{2}}}{\left(\widetilde{\xi^{2} t^{2}}\right)} .
\end{aligned}
$$

Thus, all the solutions for the Einstein-Maxwell-scalar system in arbitrary dimension either spherically symmetric $(\lambda=1)$ or "hyperbolically" symmetric $(\lambda=-1)$ is generated from the spherically symmetric solutions in four dimensions by reversing the transformation (3.16)-(3.18).

\section{EXACT SOLUTIONS FOR ARBITRARY DIMENSION AND $\lambda$}

The general solution for the $n=2, \lambda=1$, i.e. for spherically symmetric four-dimensional Einstein-Maxwell-scalar field equations is well known [17]. In our formalism, it reads as follows:

$$
\widetilde{\xi}=2 h, \quad\left(\widetilde{\xi^{2} t^{2}}\right)=-\frac{1}{g}\left(\frac{d h}{d r}\right)^{2}, \quad \widetilde{B^{2}}=-\left(\widetilde{\xi^{2} t^{2}}\right) f
$$

where the functions $f=f(r), g=g(r), h=h(r)$ are given by

$$
f=\frac{\left(x^{2}-1\right)^{2}}{\mathcal{D}^{2}}, \quad g=\mathcal{D}^{2}, \quad h=\mathcal{D}^{2} r^{2}
$$


Here the function

$$
\mathcal{D}(x):=\frac{k^{2}(x+1)^{2 \mu}-(x-1)^{2 \mu}}{\left(x^{2}-1\right)^{\mu-1}}
$$

depends on $r$ via the auxiliary variable

$$
x:=-\frac{M}{r},
$$

and $M, k^{2}$ and $\mu$ are arbitrary integration constants. The main properties of the function $\mathcal{D}(x)$ can be seen from the differential identities it satisfies:

$$
\begin{aligned}
2 \frac{d}{d x}\left(\frac{1}{\mathcal{D}} \frac{d \mathcal{D}}{d x}\right)+\left(\frac{1}{\mathcal{D}} \frac{d \mathcal{D}}{d x}\right)^{2} & =-\frac{16 \mu^{2} k^{2}}{\mathcal{D}^{2}}-\frac{4\left(1-\mu^{2}\right)}{\left(x^{2}-1\right)^{2}} \\
\left(\frac{1}{\mathcal{D}} \frac{d \mathcal{D}}{d x}\right)^{2}+\frac{4 x}{x^{2}-1}\left(\frac{1}{x}-\frac{1}{\mathcal{D}} \frac{d \mathcal{D}}{d x}\right) & =\frac{16 \mu^{2} k^{2}}{\mathcal{D}^{2}}-\frac{4\left(1-\mu^{2}\right)}{\left(x^{2}-1\right)^{2}}
\end{aligned}
$$

Using (4.1)-(4.6) in (3.19)-(3.20), we find explicitly the integration constants for a general solution with an arbitrary $n$ and $\lambda$ :

$$
\begin{aligned}
Q^{2} & =16 n(n-1) M^{2} \mu^{2} k^{2} \lambda, \\
c_{0}^{2} & =16 n(n-1) M^{2}\left(1-\mu^{2}\right) .
\end{aligned}
$$

Substituting (4.1) into (3.7), (3.9), (3.4) and (2.1), one obtains the metric:

$$
g=-\lambda f d \tau^{2}+\frac{g h^{-\left(\frac{n-2}{n-1}\right)}}{\lambda(n-1)^{2}} d r^{2}+h^{\frac{1}{n-1}} d \Omega_{\lambda}^{2},
$$

where $d \Omega_{\lambda}^{2}$ is the line element on the $n$-dimensional space of constant curvature $\lambda$.

It is worthwhile to note that the equation (4.7) demands that for the spherical symmetry $(\lambda=1)$ we take $k^{2} \geq 0$, whereas for the "hyperboloidal" symmetry $(\lambda=-1)$ one should take $k^{2} \leq 0$

The scalar field is obtained after some algebra from the first integral (3.11),

$$
\phi=\sqrt{\left(1-\mu^{2}\right)\left(\frac{n}{n-1}\right)} \log \left|\frac{x-1}{x+1}\right| .
$$

As for the Maxwell field, the electromagnetic potential, $A=A_{0} d \tau$, is obtained form (3.10) in the form: 


$$
A_{0}=-\sqrt{\lambda k^{2}\left(\frac{n}{n-1}\right)} \frac{1}{1-k^{2}} \frac{(x+1)^{2 \mu}-(x-1)^{2 \mu}}{k^{2}(x+1)^{2 \mu}-(x-1)^{2 \mu}}
$$

for the case when $k^{2} \neq 1$. When $k^{2}=1$, we have

$$
A_{0}=-\sqrt{\lambda k^{2}\left(\frac{n}{n-1}\right)} \frac{(x+1)^{2 \mu}}{(x+1)^{2 \mu}-(x-1)^{2 \mu}} .
$$

However, both potentials give rise to the same Maxwell field strength two-form

$$
F=d A=4 M \mu \sqrt{\lambda k^{2}\left(\frac{n}{n-1}\right)} \frac{x^{2}-1}{h} d \tau \wedge d r
$$

which is valid for all possible values of $k^{2}$. A straightforward check shows that $\# F=-Q \stackrel{(n)}{\eta}$, where $\stackrel{(n)}{\eta}$ is the volume $n$-form of the internal space. Thus indeed the integration constant $Q$ is the electric charge of the solution.

For completeness, let us give the dilaton field explicitly:

$$
\Phi=-\frac{1}{4}\left(\frac{n}{n-1}\right) \log h
$$

\section{DISCUSSION}

It is instructive to compare our solutions to the higher-dimensional black holes in the Brans-Dicke-Maxwell theory. The corresponding Lagrangian can be written (with the help of a suitable conformal transformation and field redefinitions) as

$$
L=-\frac{1}{2} \mathcal{R}^{A B} \wedge \eta_{A B}-\frac{1}{2} e^{-b \phi} F \wedge \# F-\frac{1}{2} d \phi \wedge \# d \phi
$$

with some constant $b$. When $b=0$, this reduces to the Lagrangian (2.4). The direct investigation shows that the condition $b \neq 0$ is crucial for the existence of non-trivial black hole solutions: the exact configurations were found in [9, 12]. They, however, cannot be used for obtaining the solutions of (2.4) from that of (5.1) by taking the limit $b \rightarrow 0$ in these configurations. The resulting solutions have trivial (constant) scalar field. In contrast, our solutions are characterized by the nontrivial scalar field, in general. 
In order to analyse the physical meaning of the constant parameters $\mu, M$ and $k^{2}$, it is convenient to replace the radial coordinate $r$ by a new variable

$$
\rho=M\left(x+\frac{1}{x}\right)
$$

Then the solution is rewritten in the form:

$$
\begin{aligned}
g & =-\lambda f d \tau^{2}+\frac{h^{-\left(\frac{n-2}{n-1}\right)}}{\lambda(n-1)^{2} f} d \rho^{2}+h^{\frac{1}{n-1}} d \Omega_{\lambda}^{2}, \\
F & =4 M \mu \sqrt{\lambda k^{2}\left(\frac{n}{n-1}\right)} \frac{1}{h} d \tau \wedge d \rho, \\
\phi & =\sqrt{\frac{\left(1-\mu^{2}\right)}{4}\left(\frac{n}{n-1}\right)} \log \left|\frac{1-y}{1+y}\right| .
\end{aligned}
$$

Here $f=f(y)$ and $h=h(y)$ are given by

$$
\begin{aligned}
f & =\frac{\left(1-y^{2}\right)^{\mu}}{\left[k^{2}(1+y)^{\mu}-(1-y)^{\mu}\right]^{2}}, \\
h & =\rho^{2}\left(1-y^{2}\right)^{1-\mu}\left[k^{2}(1+y)^{\mu}-(1-y)^{\mu}\right]^{2} .
\end{aligned}
$$

in terms of the variable $y=2 M / \rho$.

Unfortunately, when $n>2$ it is impossible to find a transformation, in a closed analytic form, to the Schwarzschild type coordinates. The above transformation (5.2) brings the metric most closely to the Schwarzschild form and it is suitable for the analysis of asymptotic behaviour of the metric. Expanding the functions (5.6)-(5.7) and the equations (5.3)-(5.4) in the asymptotic spatial region in powers of $y$ (i.e., $\rho^{-1}$ ), we find for the metric

$$
g \approx-\lambda\left(1-\frac{4 M \mu\left(k^{2}+1\right)}{\widetilde{\rho}^{n-1}}\right) d \widetilde{\tau}^{2}+\lambda\left(1+\frac{4 M \mu\left(k^{2}+1\right)}{\widetilde{\rho}^{n-1}}\right) d \widetilde{\rho}^{2}+\widetilde{\rho}^{2} d \Omega_{\lambda}^{2},
$$

and for the Maxwell and scalar fields

$$
\begin{aligned}
F & \approx \frac{4 M \mu \sqrt{\lambda k^{2} n(n-1)}}{\widetilde{\rho}^{n}} d \widetilde{\tau} \wedge d \widetilde{\rho} \\
\phi & \approx-\sqrt{\left(1-\mu^{2}\right)\left(\frac{n}{n-1}\right)} \frac{2 M\left(k^{2}-1\right)}{\widetilde{\rho}^{n-1}} .
\end{aligned}
$$

Here we accompanied the expansion by the change of the asymptotic coordinates 


$$
\widetilde{\tau}=\frac{\tau}{\left(k^{2}-1\right)}, \quad \tilde{\rho}=\left[\left(k^{2}-1\right) \rho\right]^{\frac{1}{n-1}} .
$$

Recall that $\widetilde{\rho}^{1-n}$ is a fundamental solution of the Laplace equation in a $(1+n)$-dimensional space [we consider the $(2+n)$-dimensional spacetime]. The equations (5.8)-(5.9) demonstrate that total mass $m$ and electric charge $Q$ are constructed from $\mu, M, k^{2}$ as follows:

$$
m=2 M \mu \lambda\left(k^{2}+1\right), \quad Q=4 M \mu \sqrt{\lambda k^{2} n(n-1)} .
$$

The latter relation is in complete agreement with (4.7). Note that for the spherical symmetry the electric charge and the mass always satisfy the inequality $Q / m \leq \sqrt{n(n-1)}$. Equality corresponds to the extremal charged solution. For obvious reasons, the combination $\sigma=-2 M\left(k^{2}-1\right) \sqrt{\left(1-\mu^{2}\right)\left(\frac{n}{n-1}\right)}$ can be called a scalar charge of the solution. This interpretation conforms with (4.8).

As one notices, the above identifications of integration constants are only valid when $k^{2} \neq 1$. Besides that, there are several other special cases to which our general solutions reduce for particular values of the constant parameters $\mu$ and $k^{2}$. These cases should be considered separately. In particular, from (5.3)-(5.7) it is straightforward to see that the choice $\mu= \pm 1$ yields the charged black hole configurations which extend the ReissnerNordstrom solution to a $(2+n)$-dimensional spacetime. The scalar field vanishes for these solutions. One more transformation of the radial coordinate, $R=h^{\frac{1}{2(n-1)}}$, or explicitly, $R=\left[\left(k^{2}-1\right) \rho+2 M\left(k^{2}+1\right)^{2}\right]^{\frac{1}{n-1}}$, brings the gravitational and Maxwell fields to:

$$
\begin{aligned}
g & =-f d \widetilde{\tau}^{2}+\frac{d R^{2}}{f}+R^{2} d \Omega_{\lambda}^{2}, \quad \text { with } f=\lambda-\frac{2 m}{R^{n-1}}+\frac{Q^{2}}{n(n-1) R^{2 n-2}}, \\
F & =\frac{Q}{R^{n}} d \widetilde{\tau} \wedge d R .
\end{aligned}
$$

Here $m$ and $Q$ are given by (5.12), and $\widetilde{\tau}$ is defined by (5.11). This is the higher dimensional and topological generalization of the standard Reissner-Nordstrom solution [13,5].

As we already repeatedly mentioned, the case $k^{2}=1$ requires special analysis. For $k^{2}=1$ and $\mu= \pm 1$, in the case of the spherical symmetry $(\lambda=1)$, we find from (5.3) a higher dimensional generalization of the Bertotti-Robinson solution [18. It is described by the constant electromagnetic field $F=(4 M)^{-1} \sqrt{\frac{n}{n-1}} d \tau \wedge d \rho$ and the metric 


$$
g=-\left(\rho^{2}-4 M^{2}\right) d \tau^{2}+(4 M)^{-\left(\frac{2}{n-1}\right)}\left(\frac{d \rho^{2}}{(n-1)^{2}\left(\rho^{2}-4 M^{2}\right)}+d \Omega_{\lambda}^{2}\right)
$$

of a direct product of the two-dimensional de Sitter spacetime and an $n$-sphere. Such a configuration is everywhere regular. The situation is however more complicated when $k^{2}=1$ but $\mu \neq \pm 1$. Then the electromagnetic field and the metric are only approaching the constant Bertotti-Robinson configuration (5.15) in the limit of large $r \rightarrow \infty$. But both the curvature and the electromagnetic invariants have singularities at finite values of $\rho$ for which $h=0$. This is a direct consequence of the presence of the nontrivial scalar field. Such solutions can be called higher dimensional asymptotically Bertotti-Robinson spacetimes.

Another special case which deserves being mentioned, is $\mu=0$. Then from (5.6)-(5.7) one finds $f=\left(k^{2}-1\right)^{-2}$ and $h=\left(k^{2}-1\right)^{2} \rho^{2}\left(1-y^{2}\right)$ (thus one has to take $k^{2} \neq 1$ ), and the metric reduces to [denoting $\rho_{0}=2 M\left(k^{2}-1\right)$ ]

$$
g=-d \widetilde{\tau}^{2}+\frac{\left(\widetilde{\rho}^{2}-\rho_{0}^{2}\right)^{-\left(\frac{n-2}{n-1}\right)}}{(n-1)^{2}} d \widetilde{\rho}^{2}+\left(\widetilde{\rho}^{2}-\rho_{0}^{2}\right)^{\frac{1}{n-1}} d \Omega_{\lambda}^{2}
$$

According to the above analysis, this solution has no mass and electric charge, see (5.12), but the scalar charge is nontrivial. Such a configuration represents a higher dimensional generalization of the static Roberts solution [19]. Recently an interesting study [20 of nonstatic extensions of the Roberts solution revealed, in any dimension $n$, the continuously self-similar solutions which describe collapse of scalar field with critical behaviour.

\section{ACKNOWLEDGEMENTS}

The authors are grateful to TUBITAK for the support of this research. YNO is also grateful to the Department of Physics, Middle East Technical University, for the warm hospitality. 


\section{REFERENCES}

[1] M.J. Duff, B.E.W. Nilsson, and C.N. Pope, Phys. Rept. 130 (1986) 1; J.D. Brown, Lower dimensional gravity (World Scientific: Singapore, 1988) 152 p.; S. Carlip, Quantum gravity in 2+1 dimensions (Cambridge Univ. Press: Cambridge, 1998).

[2] R.B. Mann, in: "Proc. of Workshop on the internal structure of black holes and spacetime singularities", 29 June - 3 July 1997, Haifa, Israel. Eds. L.M. Burko and A. Ori (Inst. of Phys.: Bristol, 1997; Isr. Phys. Soc.: Jerusalem, 1997) 311-342.

[3] R.-G. Cai and Y.-Z. Zhang Phys. Rev. D54 (1996) 4891; D. Klemm, Class. Quantum Grav. 15 (1998) 3195; D. Klemm, V. Moretti, and L. Vanzo, Phys. Rev. D57 (1998) 6127.

[4] J.P.S. Lemos and V.T. Zanchin, Phys. Rev. D53 (1996) 4684; N. Kaloper, Phys. Rev. D48 (1993) 4658; W.G. Anderson and N. Kaloper, Phys. Rev. D52 (1995) 4440.

[5] R.-G. Cai, J.-Y. Ji, and K.-S. Soh, Phys. Rev. D57 (1998) 6547. R.-G. Cai and K.-S. Soh, Phys. Rev. D59 (1999) 044013;

[6] A.I. Janis, E.T. Newman, and J. Winicour, Phys. Rev. Lett. 20 (1968) 878.

[7] B.C. Xantopoulos and T.E. Dialynas, J. Math. Phys. 33 (1992) 1463.

[8] A.G. Agnese and M. La Camera, Phys. Rev. D31 (1985) 1280.

[9] G.W. Gibbons and K. Maeda, Nucl. Phys. B298 (1988) 741.

[10] S.-W. Kim and B.H Cho, Phys. Rev. D40 (1989) 4028.

[11] D. Youm, Phys. Rept. 316 (1999) 1.

[12] R.-G. Cai and Y. S. Myung, Phys. Rev. D56 (1997) 3466.

[13] R.C. Myers and M.J. Perry, Ann. Phys. (NY) 172 (1986) 304.

[14] F.W. Hehl, J.D. McCrea, E.W. Mielke, and Y. Ne'eman, Phys. Rep. 258 (1995) 1. 
[15] E.W. Mielke, F. Gronwald, Yu.N. Obukhov, R. Tresguerres, and F.W. Hehl, Phys. Rev. D48 (1993) 3648; Yu.N. Obukhov, Phys. Rev. D50 (1994) 5072; Yu.N. Obukhov, S.N. Solodukhin, and E.W. Mielke, Class. Quantum Grav. 11 (1994) 3069.

[16] Yu.N. Obukhov and F.W. Hehl, Black holes in two dimensions, in: Proceedings of 179.WE-Heraeus Seminar "Black Holes Theory and Observations", Bad Honnef, Germany, 18-20 Aug 1997, Eds. F.W. Hehl, C. Kiefer, and R.J.K. Metzler (Springer: Berlin) Lect. Notes Phys. 514 (1998) 289.

[17] T. Dereli, Phys. Lett. B161 (1985) 307; T. Dereli and A. Eriş, Doğa A9 (1985) 30; A. Eriş, J. Math. Phys. 18 (1976) 824.

[18] B. Bertotti, Phys. Rev. 116 (1959) 1331; I. Robinson, Bull. Acad. Pol. Sci. 7 (1959) 351.

[19] M.D. Roberts, Gen. Rel. Grav. 21 (1989) 907; M.D. Roberts, J. Math. Phys. 37 (1996) 4557.

[20] A.V. Frolov, Class. Quantum Grav. 16 (1999) 407.

[21] T. Dereli, Yu. N. Obukhov, On the universality of low-energy string model To appear in Class.Quantum Grav. (gr-qc/9909045) 